\title{
Comments on the Adomian Decomposition Methods applied to the KdV equation
}

\section{Mahmoud AKDI, Moulay Brahim SEDRA}

Université Ibn Tofail, Faculté des Sciences, Département de Physique, LHESIR, Kénitra, Morocco

\section{Email address:}

makerase@gmail.com(M. AKDI), SEDRA-Moulay-Brahim@univ-ibntofail.ac.ma (M. B. SEDRA)

\section{To cite this article:}

Mahmoud AKDI, Moulay Brahim SEDRA. Comments on the Adomian Decomposition Methods Applied to the KdV Equation. Applied and Computational Mathematics. Vol. 2, No. 6, 2013, pp. 137-142. doi: 10.11648/j.acm.20130206.15

Abstract: Based on previous works, especially [1] and [2], we try in the present contribution to study some new aspects of the numerical solution of the $\mathrm{KdV}$ equation through the standard Adomian Decomposition Method. The use of the multistage Adomian Decomposition Method, applied to this equation, will be presented and discussed.

Keywords: KdV Equation - Standard and Multistage Adomian Decomposition Methods

\section{The Adomian Decomposition Method (ADM)}

Consider the KdV equation:

$$
\frac{\partial u}{\partial t}+6 u \frac{\partial u}{\partial x}+\frac{\partial^{3} u}{\partial x^{3}}=0, \quad u(x, 0)=f(x)
$$

which can be rewritten as follows:

$$
\frac{\partial u}{\partial t}=-R u-6 N(u), \quad u(x, 0)=f(x)
$$

where $R=\partial^{3} / \partial x^{3} \quad$ represents the linear operator of the $\mathrm{KdV}$ equation and $N(u)=u \partial u / \partial x$ is the non-linear function. According to the ADM, the solution is expressed by:

$$
u(x, t)=\sum_{n=0}^{\infty} u_{n}(x, t)
$$

and the non-linear part:

$$
N(u)=\sum_{n=0}^{\infty} A_{n}
$$

with:

$$
A_{n}=\frac{1}{n !} \frac{d^{n}}{d \lambda^{n}}\left[N\left(\sum_{i=0}^{\infty} \lambda^{i} u_{i}\right)\right]_{\lambda=0}
$$

By integrating with respect to time and using the initial conditions we have:

$$
u(x, t)=f(x)-\int_{0}^{t}[L u+6 \cdot N(u)] d s .
$$

So,

$$
u(x, t)=f(x)-\int_{0}^{t}\left[L\left(\sum_{n=0}^{\infty} u_{n}(x, s)\right)+6 \cdot \sum_{n=0}^{\infty} A_{n}\right] d s .
$$

For the $\mathrm{KdV}$ equation, the Adomian polynomials can be expressed as follows:

$$
A_{n}=\sum_{i=0}^{n} u_{i} \frac{\partial u_{n-i}}{\partial x}
$$

This allows us to deduce $u_{n}(x, t)$, namely

$$
\left\{\begin{array}{l}
u_{0}(x, t)=f(x) \\
u_{n+1}(x, t)=-\int_{0}^{t}\left[R u_{n}+A_{n}\right] d s, \quad n \geq 0
\end{array},\right.
$$

with the following initials conditions: 


$$
u(x, 0)=\frac{1}{2} \operatorname{sech}^{2}\left(\frac{x}{2}\right)
$$

We proceed in the following to compute $A_{n}(x, t)$ and $u_{n}(x, t)$ for $n \in[0,2]$ and try to determine the approximate solution $\tilde{U}_{n}(x, t)$ using the following formula:

$$
\begin{aligned}
\tilde{U}_{n}(x, t) & =\sum_{i=0}^{n} U_{i}(x, t) \\
& =f(x)-\int_{0}^{t}\left[L\left(\sum_{i=0}^{n} u_{i}(x, s)\right)+6 \cdot \sum_{i=0}^{n} A_{i}\right] d s .
\end{aligned}
$$

\section{Study of the Discrepancy of the ADM Applied to the KdV Equation}

\subsection{Calculation of Discrepancy}

We present in this section some calculations by making comparison between the values of approximated function and the exact solution through space and time evolution. We also evaluate the percentage of the discrepancy over the

\begin{tabular}{|c|c|c|c|c|c|c|c|}
\hline $\mathbf{T}$ & $\mathbf{U}_{0}$ & $\mathbf{U}_{1}$ & $\mathbf{U}_{2}$ & $\tilde{\mathbf{U}}_{2}$ & $\mathbf{U}_{\text {Exact }}$ & $\Delta$ & $\Delta / \mathbf{U}_{\text {Exact }}$ \\
\hline 0 & 0,5 & 0 & 0 & 0,5 & 0,5 & 0 & $0,00 \%$ \\
\hline 0,1 & 0,5 & 0 & $-0,00125$ & 0,49875 & 0,49875208 & $2,08039 \mathrm{E}-06$ & $0,00 \%$ \\
\hline 0,2 & 0,5 & 0 & $-0,005$ & 0,495 & 0,495033145 & $3,31454 \mathrm{E}-05$ & $0,01 \%$ \\
\hline 0,3 & 0,5 & 0 & $-0,01125$ & 0,48875 & 0,488916623 & 0,000166623 & $0,03 \%$ \\
\hline 0,4 & 0,5 & 0 & $-0,02$ & 0,48 & 0,480521491 & 0,000521491 & $0,11 \%$ \\
\hline 0,5 & 0,5 & 0 & $-0,03125$ & 0,46875 & 0,470007424 & 0,001257424 & $0,27 \%$ \\
\hline 0,6 & 0,5 & 0 & $-0,045$ & 0,455 & 0,457568481 & 0,002568481 & $0,56 \%$ \\
\hline 0,7 & 0,5 & 0 & $-0,06125$ & 0,43875 & 0,443425747 & 0,004675747 & $1,05 \%$ \\
\hline 0,8 & 0,5 & 0 & $-0,08$ & 0,42 & 0,427819393 & 0,007819393 & $1,83 \%$ \\
\hline 0,9 & 0,5 & 0 & $-0,10125$ & 0,39875 & 0,411000615 & 0,012250615 & $2,98 \%$ \\
\hline 1 & 0,5 & 0 & $-0,125$ & 0,375 & 0,393223866 & 0,018223866 & $4,63 \%$ \\
\hline 1,1 & 0,5 & 0 & $-0,15125$ & 0,34875 & 0,374739759 & 0,025989759 & $6,94 \%$ \\
\hline 1,2 & 0,5 & 0 & $-0,18$ & 0,32 & 0,355788881 & 0,035788881 & $10,06 \%$ \\
\hline 1,3 & 0,5 & 0 & $-0,21125$ & 0,28875 & 0,336596725 & 0,047846725 & $14,21 \%$ \\
\hline 1,4 & 0,5 & 0 & $-0,245$ & 0,255 & 0,317369795 & 0,062369795 & $19,65 \%$ \\
\hline 1,5 & 0,5 & 0 & $-0,28125$ & 0,21875 & 0,298292904 & 0,079542904 & $26,67 \%$ \\
\hline 1,6 & 0,5 & 0 & $-0,32$ & 0,18 & 0,279527584 & 0,099527584 & $35,61 \%$ \\
\hline 1,7 & 0,5 & 0 & $-0,36125$ & 0,13875 & 0,261211494 & 0,122461494 & $46,88 \%$ \\
\hline 1,8 & 0,5 & 0 & $-0,405$ & 0,095 & 0,243458681 & 0,148458681 & $60,98 \%$ \\
\hline 1,9 & 0,5 & 0 & $-0,45125$ & 0,04875 & 0,226360519 & 0,177610519 & $78,46 \%$ \\
\hline 2 & 0,5 & 0 & $-0,5$ & 0 & 0,209987171 & 0,209987171 & $100,00 \%$ \\
\hline
\end{tabular}
exact solution.

Table 1: Discrepancy calculation at $X=0$

Table 2: Discrepancy calculation at $X=0,5$

\begin{tabular}{cccccccc}
\hline $\mathbf{T}$ & $\mathbf{U}_{\mathbf{0}}$ & $\mathbf{U}_{\mathbf{1}}$ & $\mathbf{U}_{\mathbf{2}}$ & $\tilde{\mathbf{U}}_{\mathbf{2}}$ & $\mathbf{U}_{\text {Exact }}$ & $\boldsymbol{\Delta}$ & $\boldsymbol{\Delta} / \mathbf{U}_{\text {Exact }}$ \\
\hline 0 & 0,470007424 & 0 & 0 & 0,470007424 & 0,470007424 & 0 & $0,00 \%$ \\
0,1 & 0,470007424 & 0,011511359 & $-0,000963568$ & 0,480555216 & 0,480521491 & $-3,37243 \mathrm{E}-05$ & $-0,01 \%$ \\
0,2 & 0,470007424 & 0,023022718 & $-0,00385427$ & 0,489175872 & 0,488916623 & $-0,000259249$ & $-0,05 \%$ \\
0,3 & 0,470007424 & 0,034534077 & $-0,008672108$ & 0,495869393 & 0,495033145 & $-0,000836248$ & $-0,17 \%$ \\
0,4 & 0,470007424 & 0,046045436 & $-0,015417081$ & 0,500635779 & 0,49875208 & $-0,001883699$ & $-0,38 \%$ \\
0,5 & 0,470007424 & 0,057556795 & $-0,024089189$ & 0,50347503 & 0,5 & $-0,00347503$ & $-0,70 \%$ \\
0,6 & 0,470007424 & 0,069068154 & $-0,034688432$ & 0,504387146 & 0,49875208 & $-0,005635066$ & $-1,13 \%$ \\
\hline
\end{tabular}




\begin{tabular}{cccccccc}
\hline $\mathbf{T}$ & $\mathbf{U}_{\mathbf{0}}$ & $\mathbf{U}_{\mathbf{1}}$ & $\mathbf{U}_{\mathbf{2}}$ & $\tilde{\mathbf{U}}_{2}$ & $\mathbf{U}_{\text {Exact }}$ & $\boldsymbol{\Delta}$ & $\boldsymbol{\Delta} / \mathbf{U}_{\text {Exact }}$ \\
\hline 0,7 & 0,470007424 & 0,080579513 & $-0,047214811$ & 0,503372127 & 0,495033145 & $-0,008338981$ & $-1,68 \%$ \\
0,8 & 0,470007424 & 0,092090872 & $-0,061668324$ & 0,500429972 & 0,488916623 & $-0,011513349$ & $-2,35 \%$ \\
0,9 & 0,470007424 & 0,103602231 & $-0,078048973$ & 0,495560683 & 0,480521491 & $-0,015039191$ & $-3,13 \%$ \\
1 & 0,470007424 & 0,11511359 & $-0,096356756$ & 0,488764258 & 0,470007424 & $-0,018756833$ & $-3,99 \%$ \\
1,1 & 0,470007424 & 0,126624949 & $-0,116591675$ & 0,480040698 & 0,457568481 & $-0,022472217$ & $-4,91 \%$ \\
1,2 & 0,470007424 & 0,138136308 & $-0,138753729$ & 0,469390003 & 0,443425747 & $-0,025964256$ & $-5,86 \%$ \\
1,3 & 0,470007424 & 0,149647667 & $-0,162842918$ & 0,456812173 & 0,427819393 & $-0,02899278$ & $-6,78 \%$ \\
1,4 & 0,470007424 & 0,161159026 & $-0,188859242$ & 0,442307208 & 0,411000615 & $-0,031306593$ & $-7,62 \%$ \\
1,5 & 0,470007424 & 0,172670385 & $-0,216802702$ & 0,425875107 & 0,393223866 & $-0,032651241$ & $-8,30 \%$ \\
1,6 & 0,470007424 & 0,184181744 & $-0,246673296$ & 0,407515872 & 0,374739759 & $-0,032776113$ & $-8,75 \%$ \\
1,7 & 0,470007424 & 0,195693102 & $-0,278471026$ & 0,387229501 & 0,355788881 & $-0,03144062$ & $-8,84 \%$ \\
1,8 & 0,470007424 & 0,207204461 & $-0,31219589$ & 0,365015995 & 0,336596725 & $-0,028419271$ & $-8,44 \%$ \\
1,9 & 0,470007424 & 0,21871582 & $-0,34784789$ & 0,340875355 & 0,317369795 & $-0,02350556$ & $-7,41 \%$ \\
2 & 0,470007424 & 0,230227179 & $-0,385427025$ & 0,314807579 & 0,298292904 & $-0,016514675$ & $-5,54 \%$ \\
\hline
\end{tabular}

Table 3: Discrepancy calculation at $X=1$

\begin{tabular}{|c|c|c|c|c|c|c|c|}
\hline $\mathbf{T}$ & $\mathbf{U}_{0}$ & $\mathbf{U}_{1}$ & $\mathbf{U}_{2}$ & $\tilde{\mathbf{U}}_{2}$ & $\mathbf{U}_{\text {Exact }}$ & $\Delta$ & $\boldsymbol{\Delta} / \mathbf{U}_{\text {Exact }}$ \\
\hline 0 & 0,393223866 & 0 & 0 & 0,393223866 & 0,393223866 & 0 & $0,00 \%$ \\
\hline 0,1 & 0,393223866 & 0,01817155 & $-0,000353256$ & 0,41104216 & 0,411000615 & $-4,15455 \mathrm{E}-05$ & $-0,01 \%$ \\
\hline 0,2 & 0,393223866 & 0,036343099 & $-0,001413023$ & 0,428153942 & 0,427819393 & $-0,000334549$ & $-0,08 \%$ \\
\hline 0,3 & 0,393223866 & 0,054514649 & $-0,003179302$ & 0,444559213 & 0,443425747 & $-0,001133466$ & $-0,26 \%$ \\
\hline 0,4 & 0,393223866 & 0,072686198 & $-0,005652093$ & 0,460257972 & 0,457568481 & $-0,002689491$ & $-0,59 \%$ \\
\hline 0,5 & 0,393223866 & 0,090857748 & $-0,008831395$ & 0,475250219 & 0,470007424 & $-0,005242795$ & $-1,12 \%$ \\
\hline 0,6 & 0,393223866 & 0,109029297 & $-0,012717209$ & 0,489535955 & 0,480521491 & $-0,009014463$ & $-1,88 \%$ \\
\hline 0,7 & 0,393223866 & 0,127200847 & $-0,017309534$ & 0,503115179 & 0,488916623 & $-0,014198555$ & $-2,90 \%$ \\
\hline 0,8 & 0,393223866 & 0,145372396 & $-0,022608372$ & 0,515987891 & 0,495033145 & $-0,020954746$ & $-4,23 \%$ \\
\hline 0,9 & 0,393223866 & 0,163543946 & $-0,02861372$ & 0,528154092 & 0,49875208 & $-0,029402012$ & $-5,90 \%$ \\
\hline 1 & 0,393223866 & 0,181715495 & $-0,035325581$ & 0,539613781 & 0,5 & $-0,039613781$ & $-7,92 \%$ \\
\hline 1,1 & 0,393223866 & 0,199887045 & $-0,042743952$ & 0,550366959 & 0,49875208 & $-0,051614879$ & $-10,35 \%$ \\
\hline 1,2 & 0,393223866 & 0,218058594 & $-0,050868836$ & 0,560413625 & 0,495033145 & $-0,06538048$ & $-13,21 \%$ \\
\hline 1,3 & 0,393223866 & 0,236230144 & $-0,059700231$ & 0,569753779 & 0,488916623 & $-0,080837156$ & $-16,53 \%$ \\
\hline 1,4 & 0,393223866 & 0,254401693 & $-0,069238138$ & 0,578387422 & 0,480521491 & $-0,097865931$ & $-20,37 \%$ \\
\hline 1,5 & 0,393223866 & 0,272573243 & $-0,079482556$ & 0,586314553 & 0,470007424 & $-0,116307129$ & $-24,75 \%$ \\
\hline 1,6 & 0,393223866 & 0,290744793 & $-0,090433486$ & 0,593535173 & 0,457568481 & $-0,135966692$ & $-29,72 \%$ \\
\hline 1,7 & 0,393223866 & 0,308916342 & $-0,102090928$ & 0,600049281 & 0,443425747 & $-0,156623534$ & $-35,32 \%$ \\
\hline 1,8 & 0,393223866 & 0,327087892 & $-0,114454881$ & 0,605856877 & 0,427819393 & $-0,178037484$ & $-41,62 \%$ \\
\hline 1,9 & 0,393223866 & 0,345259441 & $-0,127525346$ & 0,610957962 & 0,411000615 & $-0,199957347$ & $-48,65 \%$ \\
\hline 2 & 0,393223866 & 0,363430991 & $-0,141302322$ & 0,615352535 & 0,393223866 & $-0,222128669$ & $-56,49 \%$ \\
\hline
\end{tabular}

As we can easily observe, the convergence is limited relatively to the time parameter, and it's shown that it diverge consistently beyond the value of $\tau_{0}[1]$ defining the time limit of convergence. 


\subsection{Determination of the Contribution of Each Element of the Adomian Polynomial}

In order to obtain a behavior of the evolving manner of the elements of the Adomian polynomial, we elaborate the calculation for $x=1$, to precise the percentage of each one separately, as exposed in the following table:

Table 4: The evolving of Ui

\begin{tabular}{|c|c|c|c|c|c|c|}
\hline $\mathbf{T}$ & $\mathbf{U}_{0}$ & $\mathrm{U}_{1}$ & $\mathbf{U}_{2}$ & $\mathbf{U}_{3}$ & $\mathbf{U}_{4}$ & $\mathbf{U}_{5}$ \\
\hline 0 & 1 & $0,00 \%$ & $0,00 \%$ & $0,00 \%$ & $0,00 \%$ & $0,00 \%$ \\
\hline 0,1 & $95,66 \%$ & $4,42 \%$ & $-0,09 \%$ & $0,01 \%$ & $0,00 \%$ & $0,00 \%$ \\
\hline 0,2 & $91,80 \%$ & $8,48 \%$ & $-0,36 \%$ & $0,05 \%$ & $0,00 \%$ & $0,00 \%$ \\
\hline 0,3 & $88,33 \%$ & $12,25 \%$ & $-0,77 \%$ & $0,16 \%$ & $-0,01 \%$ & $0,00 \%$ \\
\hline 0,4 & $85,16 \%$ & $15,74 \%$ & $-1,22 \%$ & $0,34 \%$ & $-0,02 \%$ & $0,01 \%$ \\
\hline 0,5 & $82,23 \%$ & $19,00 \%$ & $-1,85 \%$ & $0,64 \%$ & $-0,06 \%$ & $0,03 \%$ \\
\hline 0,6 & $79,50 \%$ & $22,04 \%$ & $-2,57 \%$ & $1,06 \%$ & $-0,11 \%$ & $0,07 \%$ \\
\hline 0,7 & $76,92 \%$ & $24,88 \%$ & $-3,39 \%$ & $1,63 \%$ & $-0,20 \%$ & $0,16 \%$ \\
\hline 0,8 & $74,44 \%$ & $27,52 \%$ & $-4,28 \%$ & $2,36 \%$ & $-0,33 \%$ & $0,29 \%$ \\
\hline 0,9 & $72,03 \%$ & $29,96 \%$ & $-5,24 \%$ & $3,25 \%$ & $-0,51 \%$ & $0,51 \%$ \\
\hline 1 & $69,67 \%$ & $32,20 \%$ & $-6,26 \%$ & $4,31 \%$ & $-0,75 \%$ & $0,84 \%$ \\
\hline 1,1 & $67,32 \%$ & $34,22 \%$ & $-7,32 \%$ & $5,54 \%$ & $-1,07 \%$ & $1,30 \%$ \\
\hline 1,2 & $64,95 \%$ & $36,02 \%$ & $-8,40 \%$ & $6,95 \%$ & $-1,46 \%$ & $1,94 \%$ \\
\hline 1,3 & $62,56 \%$ & $37,58 \%$ & $-9,50 \%$ & $8,50 \%$ & $-1,93 \%$ & $2,79 \%$ \\
\hline 1,4 & $60,11 \%$ & $38,89 \%$ & $-10,58 \%$ & $10,21 \%$ & $-2,50 \%$ & $3,88 \%$ \\
\hline 1,5 & $57,59 \%$ & $39,92 \%$ & $-11,64 \%$ & $12,03 \%$ & $-3,15 \%$ & $5,25 \%$ \\
\hline 1,6 & $55,01 \%$ & $40,67 \%$ & $-12,65 \%$ & $13,94 \%$ & $-3,90 \%$ & $6,93 \%$ \\
\hline 1,7 & $52,35 \%$ & $41,13 \%$ & $-13,59 \%$ & $15,91 \%$ & $-4,73 \%$ & $8,93 \%$ \\
\hline 1,8 & $49,63 \%$ & $41,28 \%$ & $-14,44 \%$ & $17,91 \%$ & $-5,64 \%$ & $11,27 \%$ \\
\hline 1,9 & $46,85 \%$ & $41,13 \%$ & $-15,19 \%$ & $19,88 \%$ & $-6,60 \%$ & $13,94 \%$ \\
\hline 2 & $44,03 \%$ & $40,69 \%$ & $-15,82 \%$ & $21,79 \%$ & $-7,62 \%$ & $16,93 \%$ \\
\hline
\end{tabular}

\subsection{Interpretation of the Results}

As described in the previous table, the contribution of the first elements of $U_{i}$, is more important at the beginning, while we progress in the time calculation, and it gradually decreases in favor of the other $U_{i}$ elements. The $U_{i}$ are evolving in positive and negative values and progressively their sum $\tilde{U}$ diverges from the exact solution.

So, the divergence cannot be attributed to special categories of $U_{i}$, which we can avoid by implementing a filter, but it is a mass fact. From where the interest to develop other technique, that allow us to overcome the limitations of the standard ADM.

\section{Multistage Adomian Decomposition Method (MS-ADM) for KdV Equation}

\subsection{Principe of the Method}

To simplify, we present the MS-ADM by the following diagram:

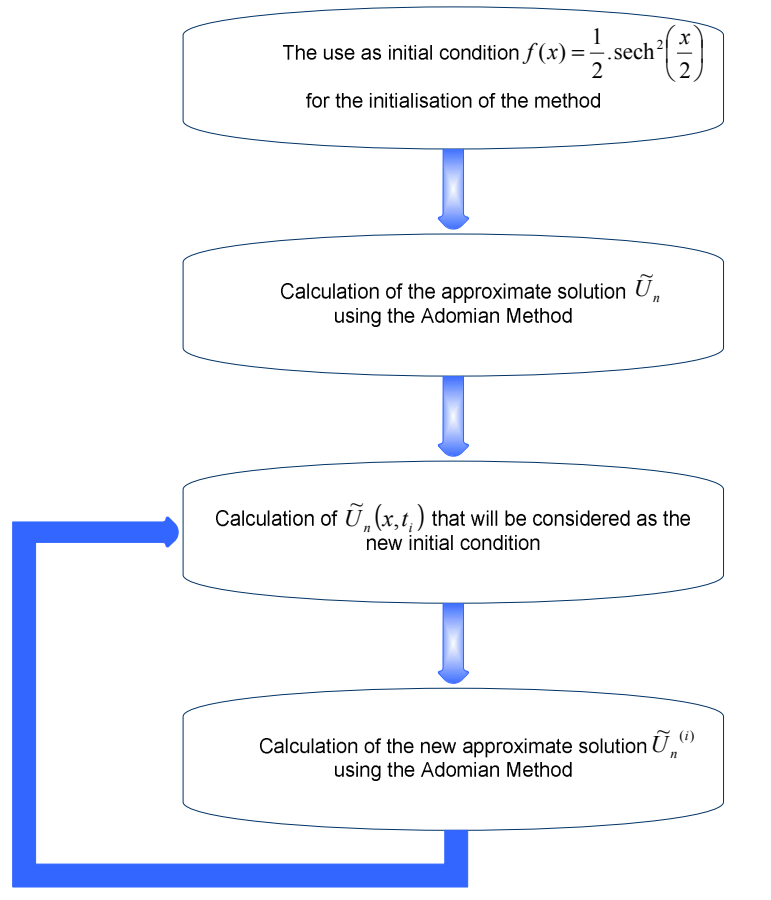


Based on this principle, we can write

$$
f(x)=\left.\tilde{U}_{2}(x, t)\right|_{t_{0}=0,1}
$$

Hence, after calculation we found:

$$
f(x)=2,21 \cdot e^{x} \cdot \frac{\left(e^{2 x}+1,79186 \cdot e^{x}+0,819005\right)}{\left(e^{x}+1\right)^{4}}
$$

Using the previous backgrounds, we have to recalculate the approximated function $\tilde{U}_{2}^{*}(x, t)$ based on the new expression of the initial condition $f(x)$, for: $t=t_{2}$.

As made before for the standard Adomian decomposition method and also taking into account the new formulation, we have to precise the following expressions:

$$
A_{0}^{*}=U_{0}^{*} \frac{\partial U_{0}^{*}}{\partial x}
$$

$$
\begin{aligned}
& U_{1}^{*}=-\int_{0}^{t}\left[R U_{0}^{*}+A_{0}^{*}\right] d s ; \\
& A_{1}^{*}=U_{0}^{*} \frac{\partial U_{1}^{*}}{\partial x}+U_{1}^{*} \frac{\partial U_{0}^{*}}{\partial x} ; \\
& U_{2}^{*}=-\int_{0}^{t}\left[R U_{1}^{*}+A_{1}^{*}\right] d s .
\end{aligned}
$$

\subsection{Discrepancy Study}

After calculation for $t_{2}=0,15$, and in comparison with the standard Adomian decomposition method, we present the following results in the table below:

Table5: Discrepancies calculation

\begin{tabular}{cccccc}
\hline & \multicolumn{5}{c}{ Values of $\mathbf{~}$} \\
\hline & $\mathbf{0}$ & $\mathbf{0 , 2 5}$ & $\mathbf{0 , 5}$ & $\mathbf{0 , 7 5}$ & $\mathbf{1}$ \\
\hline Exact Solution & 0,4971980 & 0,4987521 & 0,4849948 & 0,4575685 & 0,4195456 \\
ADM & 0,4971875 & 0,4988099 & 0,4851064 & 0,4577083 & 0,4196864 \\
S/ADM & 0,0000105 & $-0,0000578$ & $-0,0001116$ & $-0,0001399$ & $-0,0001407$ \\
MS-ADM & 0,4881485 & 0,4734446 & 0,4525302 & 0,4256124 & 0,3918466 \\
$\Delta$ MS-ADM & 0,0090495 & 0,0253075 & 0,0324646 & 0,0319561 & 0,0276990 \\
\hline
\end{tabular}

For establishing a better evaluation of these results, we will elaborate a graphical representation of these functions:

- $f_{1}$ : the exact solution;

- $f_{2}$ : approximate solution using the standard Adomian decomposition method;

- $f_{3}$ : approximate solution using the Multistage Adomian decomposition method.

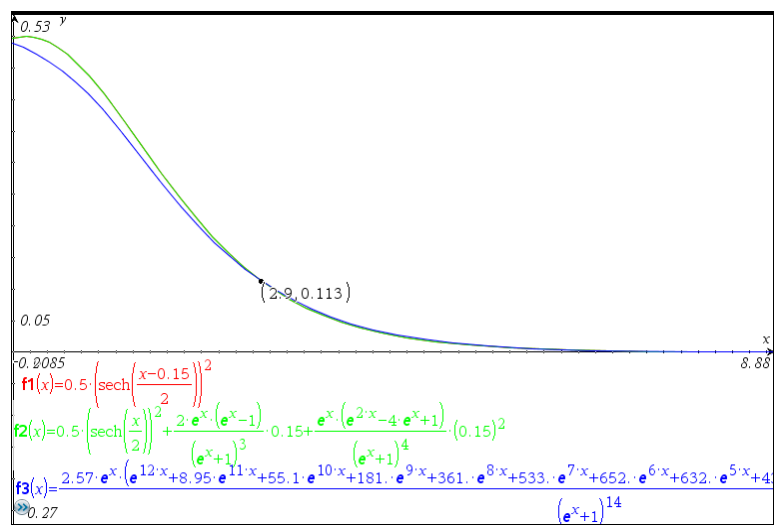

Graph 1: Comparative graphical representations
And for further richest visualization, we plot also these function:

$$
\left\{\begin{array}{l}
F_{\Delta / A D M}=U_{E x a c t}-\tilde{U}_{A D M} \\
F_{\Delta / M S-A D M}=U_{E x a c t}-\tilde{U}_{M S-A D M}
\end{array}\right.
$$

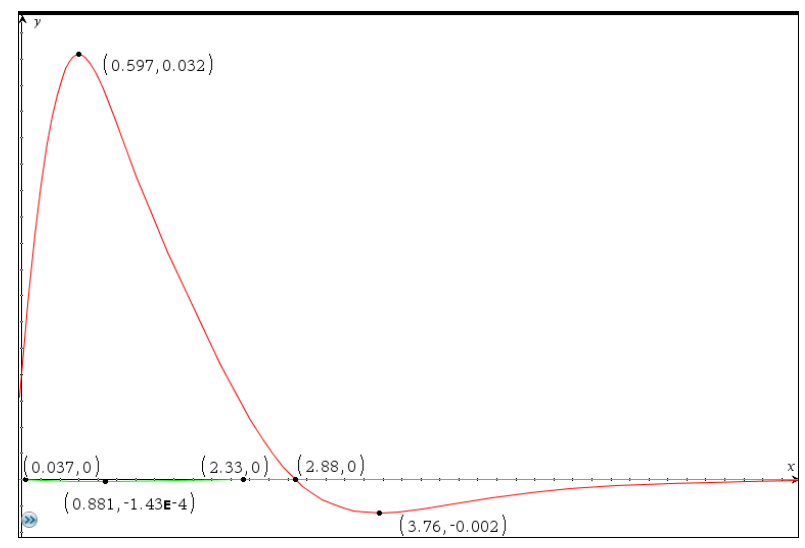

Graph 2: Discrepancies representation 
Where:

- $F_{\triangle / A D M}$ : is represented by the green color ;

- $F_{\triangle / M S-A D M}$ : is represented by the red color.

\subsection{Discussion}

Based on the previous numerical results and plotting graphical representations, we can deduce the following results:

1 Asymptotically, the both approximated functions converge to the exact solution for this time value;

2 The discrepancy graphic for $F_{\triangle / M S-A D M}$, show that this function evolve between $+0,032$ and $-0,002$ and have single null value at $x=2,88$ and after that conserve a negative value;

3 The discrepancy graphic for $F_{\triangle / A D M}$, show that this function have two null values for $x=0,037$ and $x=2,33$ and minimum for $x=0,881$, which it means that this function conserve after his second zero, a positive value.

Therefore, the both approximated functions are reaching the exact solution, but not by the same manner. The positive evolution for the function $F_{\triangle / A D M}$, lead for the next estimation processes of calculation of the Adomian decomposition method, to the amplification of the approximation error, contributing to it divergence.

Contrariwise, the Multistage Adomian decomposition method have a more important positive discrepancy at the beginning (in comparison with the Adomian decomposition method), but it becomes negative for the rest of the spatial parameter, while remaining near of 0 .

\section{Conclusion}

Through this work, we were able to evolve with precision the nature and the progression of the discrepancy between the exact solution of $\mathrm{KdV}$ equation and the approximated one using the standard Adomian decomposition method.

We have also, applied the Multistage Adomian decomposition method to the resolution of the KdV equation, which it allows us to elaborate a non-divergent method with an acceptable accuracy level. Hence, the amplification of the approximation error, as it occurs for the standard Adomian decomposition method, is avoided.

\section{Acknowledgements}

The authors would like to express their thanks to Pr. Saad CHOUKRI (EMI) for useful conversations.

\section{References}

[1] M.Akdi, M. B. Sedra, "Numerical KDV equation by the Adomian decomposition method", American Journal of Modern Physics. (2013)

[2] M. Akdi, "Study of solitary waves: results of resolution by numerical methods of the Korteweg-de Vries equation and simulation of the fluid flow", Ph. D. Thesis. (2013)

[3] M. Inc, On numerical solution of partial differential equation by the decomposition method, Kragujevac J Math. (2004)

[4] García-Olivares, A., "Analytic solution of partial differential equations with Adomian's decomposition", Kybernetes (Bingley, U.K.: Emerald) 32: 354-368. (2003)

[5] García-Olivares, A., "Analytical solution of nonlinear partial differential equations of physics", Kybernetes (Bingley, U.K. Emerald) 32: 548-560. (2003)

[6] Liao, S.J., "Homotopy Analysis Method in Nonlinear Differential Equation", Berlin \& Beijing: Springer \& Higher Education Press. (2012)

[7] G. Adomian, "Solving Frontier Problems of Physics: The Decomposition Method", Kluwer, Dordrecht. (1994)

[8] K. Abbaoui and Y. Cherruault, Convergence of adomian's method applied to differential equations, Comp Math Appl. (1994)

[9] K. Abbaoui and Y. Cherruault, Convergence of Adomian's method applied to nonlinear equations, Math Computer Model. (1994)

[10] N. Himoun, K. Abbaoui, and Y. Cherruault, New results of convergence of Adomian's method,Kybernetes. (1999)

[11] J. Biazar, E. Babolian, A. Nouri, R. Islam, "An alternate algorithm for computing Adomian decomposition method in special cases", Appl. Math. Comput. 138, 1-7. (2003)

[12] J. Biazar, M. Tango, E. Babolian, R. Islam, "Solution of the kinetic modeling of lactic acid fermentation using Adomian decomposition method", Appl. Math. Comput. 139, 249-258. (2003)

[13] Kolebaje O. T., Ojo O. L., Akinyemi P., Adenodi R. A., "On the application of the multistage laplace adomian decomposition method with pade approximation to the rabinovich-fabrikant system", Advances in Applied Science Research. (2013)

[14] Nur Isnida Razali, M.S.H Chowdhury, Waqar Asrar, "The Multistage Adomian Decomposition Method for Solving Chaotic Lü System", Middle-East Journal of Scientific Research. (2013) 